\title{
ESTRUCTURA DE LAS COMUNIDADES MACROZOOBENTÓNICAS DE LOS HUMEDALES DE VENTANILLA, CALLAO, PERÚ
}

\author{
MACROZOOBENTHIC STRUCTURE COMMUNITY OF VENTANILLA WETLANDS, \\ CALLAO, PERU
}

${ }^{1}$ Carla Vizcardo \& ${ }^{2}$ Patricia Gil-Kodaka

\begin{abstract}
Resumen
La estructura macrozoobentónica fue analizada durante el período de abril a setiembre de 2008. Se realizaron colectas mensuales en nueve estaciones de muestreo ubicadas en tres lagunas permanentes más características de los humedales de Ventanilla. La recolección de las muestras de sedimento se realizó con un cilindro de PVC $\left(0,024 \mathrm{~m}^{2}\right.$ de área). Se determinaron las variables fisicoquímicas del agua (temperatura, oxigeno disuelto, conductividad, nitratos, coliformes totales y profundidad). La comunidad estuvo compuesta por tres filos: Annelida (Clase Oligochaeta), Artrophoda (Clase Arachnoidea y Clase Hexápoda) y Mollusca (clase Gastropoda). La abundancia promedio en las tres lagunas: Laguna Mirador (LMI), Laguna Media (LME) y Laguna Mayor (LMA) fue de 79,7, 79,9 y 605,5 individuos respectivamente. La menor abundancia se observó en el mes de abril (128 ind.) y la mayor en el mes de junio (430 ind.) decreciendo hasta setiembre (141 ind.). Los taxones más abundantes fueron Hydrobia sp y Melanoides tuberculata. Los bajos valores de diversidad $\left(H^{\prime}=1,27\right)$, riqueza $(d=0,93)$ y equidad $\left(J^{\prime}=0,4\right)$ es el resultado de factores que producen estrés ambiental, como la fluctuación hidrológica en los cuerpos de agua, sumado a esto las presiones antrópicas que vienen afectando al humedal. La dinámica de los macrozoobentos presentó $r$ elación con las variables fisicoquímicas del agua.
\end{abstract}

Palabras clave: Humedales, Hydrobia, Melanoides, macrozoobentos, Perú.

\begin{abstract}
Macrozoobenthic structure was analyzed from April to September 2008. Samples were obtained monthly from nine sampling stations located in three permanent lagoons from the wetlands of Ventanilla.

Sediment samples were performed using a PVC cylinder $\left(0.024 \mathrm{~m}^{2}\right.$ area). Physicochemical water variables as temperature, dissolved oxygen, conductivity, nitrates, total coliforms and depth were determined. The community was composed of three phyla: Annelida (Class Oligochaeta), Arthropoda (Class Arachnoidea and Class Hexapoda) and Mollusca (Class Gastropoda). The average abundance in the tree lagoons Lagoon Mirador (LMI), Lagoon Media (LME) and Lagoon Mayor (LMA) was 79.7, 79.9 and 605.5 individuals respectively. The lowest abundance was observed in April (128 ind.). The highest abundance was observed in June (430 ind.), decreasing through September (141ind.). The most abundant taxa were Hydrobia sp and Melanoides tuberculata. The low values of diversity $\left(\mathrm{H}^{\prime}=1.27\right)$, richness $(\mathrm{d}=$ 0.93 ) and equity $\left(\mathrm{J}^{\prime}=0.4\right)$ is the result of environmental stress factors, such as fluctuating hydrological bodies water, added to this the anthropogenic pressures that are affecting the wetland. The dynamics of macrozoobenthos presented relation with the water physicochemical variables.
\end{abstract}

Key words: Wetlands, Hydrobia, Melanoides, macrozoobenthos, Perú.

\section{Introducción}

Los humedales comprende una gran variedad de formas y ambientes, así como cuerpos de agua temporales o permanentes que envuelven un sin fin de organismos que determinan su biología y ecología (Franco et al., 2000; Espinosa \& Morales, 2008). Estos sistemas están definidos como intermedios o de transición entre los ecosistemas acuáticos y terrestres de aguas lénticas (Cowardin et al., 1979). Son ecosistemas que han sido de las zonas más degradadas por sufrir presión antropogénica. Sin embargo, se reconoce el gran valor que estos ecosistemas juegan en la naturaleza actuando como amortiguadores, lográndose algunos avances en la conservación de estos ecosistemas sobre todo en la costa del Perú (Gopal et al., 1982; Obando et al., 1998; Pulido, 1998).

Los organismos bentónicos se encuentran íntimamente relacionados con el fondo y se han utilizado ampliamente como indicadores de contaminación y de la calidad del agua por presentar diversas características como, ciclos de vida largos, relativamente sedentarios y sensibles a diferentes concentraciones de contaminación que los hacen especiales para su estudio. Su distribución y

\footnotetext{
${ }^{1}$ Facultad de Ciencias, Universidad Nacional Agraria La Molina, Lima, Perú. E-mail: carlamilagrosvg@gmail.com

${ }^{2}$ Facultad de Pesquería, Universidad Nacional Agraria La Molina, Lima, Perú. E-mail: pgilkodaka@lamolina.edu.pe
} 
abundancia está influenciada por factores biogeográficos y características del ambiente (Iannacone et al, 2003; Alonso \& Camargo, 2005; Espinosa \& Morales, 2008; Sadegui et al, 2010; Gittenberger, 2011).

En la provincia constitucional del Callao - Perú se ubica los Humedales de Ventanilla, conformada por un conjunto de sistemas lagunares permanentes y temporales que se alimentan en condiciones naturales por filtraciones del rio Chillón y del litoral marino. Este humedal integra junto a otros humedales de la costa, una red de afloramientos que son muy importantes para la vida de muchas especies (Iannacone, 2003).

Esta Área de Conservación Regional constituye refugios, sitios de reproducción y alimentación especialmente de aves acuáticas endémicas y migratorias. A pesar de esto, los cuerpos de agua presentan una problemática por las invasiones y sobrepoblación en zona cercanas a la misma. En la actualidad existen muy pocos trabajos publicados sobre inventarios y programas de monitoreo permanente de biota en este humedal.

El objetivo de la presente investigación fue analizar la estructura de las comunidades macrozoobentonicas de 3 lagunas en los humedales de Ventanilla y su relación con los parámetros fisicoquímicos.

\section{Materiales y métodos \\ Área de estudio}

Los Humedales de Ventanilla se encuentra ubicada cerca al litoral marino en el distrito de Ventanilla, Provincia Constitucional del Callao, Perú (Figura 1), se sitúa entre las coordenadas $11^{\circ} 51^{\prime} 23^{\prime \prime}-11^{\circ} 52^{\prime} 42^{\prime \prime}$ Lat. Sur y $77^{\circ} 07^{\prime}$ $43^{\prime \prime}-77^{\circ} 09^{\prime} 32^{\prime \prime}$ Long. Oeste. El área de los humedales es de 275,8 ha, declarada "Área de Conservación Regional" en el año 2006.

Se encuentra conformada por varios cuerpos de agua permanentes y temporales, así como de áreas donde predominan especies vegetales como: Salicornia fruticosa (salicorniales), Distichlis spicata (gramadal), Typha dominguensis (totoral), Cyperus sp (juncal) y Tessaria integrifolia (Zona arbustiva); y una variedad de aves como Cigueñuelos Himantopus mexicanus, garzas Egretta alba y E. caeruela, pollas de agua Gallinula chloropus, gallaretas Fulica ardesica, patos (Alvarez y Iannacone, 2008). Los humedales se alimentan en condiciones naturales por filtraciones del rio Chillón y del litoral marino, subiendo el nivel de agua entre los meses de mayo y junio.

\section{Estaciones de muestreo}

Se determinaron un total de nueve estaciones de muestreo (Figura 1) distribuidas en tres espejos de agua permanentes, denominadas en este estudio como Laguna Mirador: LMI, Laguna Media: LME y Laguna Mayor: LMA (Tabla 1). Las estaciones fueron escogidas previo reconocimiento de campo y siguiendo las recomendaciones de Moliner y Gonzáles (2003). Las características de las estaciones se describen a partir de las observaciones realizadas por los autores:

Laguna Mirador: Estaciones E1 y E2. Es una área de Reserva Ecológica Intangible (Parque Ecológico Municipal "El Mirador") se encuentra cercada a cargo de la Municipalidad de Ventanilla, caracterizado por filtraciones de agua del río Chillón, vegetación predomínate Typha domingensis "totora" y Chara $s p$, material vegetal en descomposición, sustrato tipo fangoso, color de agua oscura y habitado por una gran variedad de aves residentes y migratorias.

Laguna Media: Estaciones E3, E4 y E5: Ubicado frente al Asentamiento Humano "Defensores de la Patria". Zona accesible a la población, frecuente para captura de peces ornamentales Poecilia reticulata "guppy" y Poecilia sp. "molly"; y preferencia de alimentación de pollas de agua, gallaretas y patos. Presenta filtraciones del río Chillón, vegetación vascular tipo "totora", Chara sp y algas, fondo de tipo arenoso y color de agua turbia. Presencia de restos platicos en el agua, afectada por perturbación antropogénica.

Laguna Mayor: Estaciones E6, E7, E8 y E9. Ubicado frente al litoral marino (balneario Costa Azul), aguas de apariencia oscura con influencia de filtración marina y del río Chillón, vegetación dominada por Salicornia fruticosa y aves por cigüeñuelas, garzas y patos. Fondo de tipo fangoso/arenoso y abundante materia orgánica en descomposición. Presencia de restos de basura por la presión antrópica que sufre, debido a su cercanía a la playa.

\section{Colección y procesamiento de las muestras}

Los muestreos fueron realizados en los meses de abril a setiembre de 2008, recolectándose muestras por triplicado en cada estación de muestreo con ayuda de un muestreador de un litro de capacidad, que consistió en un cilindro de PVC $\left(0,024 \mathrm{~m}^{2}\right.$ de área) con un mango de madera. El material colectado se guardó en frascos de plástico de un litro de capacidad debidamente rotulado y fijado con formol al 10\%.

Los macrozoobentos fueron separados utilizando un tamiz de 250 micras, preservándose en alcohol etílico al $70 \%$. Una vez preservados, los organismos de la macrofauna se identificaron bajo un microscopio estereoscópico hasta el nivel taxonómico más bajo, con ayuda de claves taxonómicas especializadas (Borror et al., 1995; Vivar et al., 1998) y consultando con especialistas. Para cada taxón se determino la abundancia, Abundancia relativa (\%) y los índices biológicos de Diversidad de Shannon - Wiener (H'), Riqueza de Margalef (d), Dominancia de Simpson (D) y Equidad de Pielou (J') (Moreno, 2001). Para verificar si existen diferencias significativas entre lagunas se utilizó el test ANOSIM de un factor (LMI x LME x LMA) con el uso del programa PRIMER 6.0 (Clarke, 1993; Anderson, 2001). 
Se tomaron muestras de agua de las lagunas para medir la temperatura $\left({ }^{\circ} \mathrm{C}\right)$ con un termómetro de mercurio; oxígeno disuelto (mg/l) por el método de Winkler "in situ”; la conductividad eléctrica $(\mathrm{mS})$ con un conductimetro, el $\mathrm{pH}$, con un pHmetro de mesa en el laboratorio. Además muestras de agua para nitratos y coliformes totales (CT) enviados a un laboratorio. Se midió también la profundidad de los cuerpos de agua.

Se aplico un análisis de componentes principales (ACP) a una matriz de correlaciones de las variables fisicoquímicas, con la finalidad de determinar los parámetros que explicaban la mayor variabilidad en las lagunas. Se utilizó un análisis de correspondencia canónica (ACC) para identificar relaciones entre las variables fisicoquímicas y la estructura de la comunidad macrozoobentónica (Cuadras, 2012). Los análisis ACP y ACC se calcularon utilizando los datos sin transformación con el programa estadístico PAST.

\section{Resultados}

\section{Factores fisicoquímicos}

El análisis de componentes principales (ACP) indica que la variación entre las variables fisicoquímicas puede ser explicado por los dos primeros componentes principales en un $70 \%$. El primer eje separa los sitios con valores elevados de CE y las temperaturas de agua y aire. El segundo eje ordena los sitios en función al OD, $\mathrm{pH}$ y profundidad (Figura 2b). De acuerdo a este análisis las tres lagunas se encuentran distribuidas dentro de la misma variabilidad ambiental debido a que las estaciones se encuentran dispersos en este diagrama de ordenación (Figura 2a), es decir, que las estaciones de las 3 lagunas comparten características ambientales similares, a excepción de la mayor CE en LMA (Figura 2).

El ACP mostró dos variables fisicoquímicos que explicaban la dinámica fisicoquímica de las lagunas (Tabla 2). En este caso, aparecen los valores propios de cada componente principal (parámetros fisicoquímicos). El OD (mg/l) en el eje 1, con un $46 \%$ de variabilidad y CE (mS), con un $24 \%$ de variabilidad en el eje 2 , son las variables que dominan y caracterizan la dinámica fisicoquímicas de las lagunas. Los demás factores $(\mathrm{pH}$, Temperaturas y profundidad) se comportaron de manera similar a través del tiempo de estudio no presentando una varianza significativa. De la Figura 3 se pueden describir dos grupos: El grupo I, caracterizado por bajos valores de $\mathrm{OD}$ y altos valores de $\mathrm{CE}$, que coincidieron en los primeros meses de muestreo de abril y mayo en estaciones de LMA, y el grupo II, caracterizado por altos valores de OD y bajos de CE, que ocurrieron en junio en estaciones de LMI y LME, y en los demás meses permanecen relativamente constantes (Tabla 3).

Tomando en cuenta los Estándares de Calidad Ambiental (ECA), DS - № 002 - 2008 - MINAM para la categoría 4, valores de OD (Abr y May) estuvieron por debajo de lo recomendado para la conservación del ambiente acuática, así como también para nitratos y coliformes totales. Estos 2 últimos parámetros no se tomaron en cuenta para ACP debido a que solo se registraron para el mes de abril, junio y setiembre (Tabla 3)

\section{Estructura comunitaria}

Se registraron 11 taxones dentro de 3 filos. El filo Mollusca fue el más representativo, donde la familia Hydrobiidae, constituido por Hydrobia sp, fue el dominante de la LMI y la LMA, con un $38,6 \%$ y $91,7 \%$ de abundancia del total de organismos respectivamente; por otro lado, la familia Thiaridae con Melanoides tuberculata, fue el dominante de la LME, con un 55,2\% de la abundancia total (Tabla 4).

Un total de 42080 organismos fueron cuantificados de 54 muestras de sedimento recolectadas. En la Tabla 4 se muestran las abundancias promedio de los macroinvertebrados bentónicos. La abundancia promedio de individuos en LMI fue de 79,7, en esta laguna Hydrobia sp presentó una abundancia promedio de 307,2 individuos. En LME fue de 79,9, donde Melanoides tuberculata presentó una abundancia de 423,5 individuos y en LMA la abundancia fue de 605,5 individuos, Hydrobia $s p$ presentó una abundancia de 4997 ind. Diferenciando entre lagunas se observa la mayor abundancia en LMA y similares entre LMI y la LME. Comparando entre meses de muestreo, menor abundancia se observó durante el mes de abril (128 ind.) y la mayor en el mes de junio (430 ind.) decreciendo hasta setiembre (141 ind.).

La diversidad (H') total para los macroinvertebrados bentónicos fue de 1,27 , para la riqueza (d) 0,93 , la equidad (J') 0,3 y la dominancia (D) 0,4. La mayor diversidad, riqueza y equidad se obtuvo en la $\operatorname{LMI}(2,60,1,48$ y 0,84 respectivamente) y el máximo valor de la dominancia se registró en la $\operatorname{LMA}(0,99)$ (Tabla 5).

Los resultados del análisis de ANOSIM a partir de los datos de abundancia detectaron diferencias significativas entre LMI, LME y LMA (ANOSIM: $\mathrm{R}=0.992, \mathrm{p}=$ 0.001). La composición faunística de las estaciones que conforman la LMI no difirió de los LME (ANOSIM: R = $1.00, \mathrm{p}=0.1$ ) y LMA (ANOSIM: $\mathrm{R}=1.00, \mathrm{p}=0.067$ ). Entre tanto, estaciones de la LME y LMA presentaron diferencias significativas entre su composición faunística (ANOSIM: $\mathrm{R}=1.00, \mathrm{p}=0.029$ ).

El Análisis de Correspondencia Canónica ACC mostrada en la Figura 4, indica que la varianza acumulada de la relación entre las variables fisicoquímicos y biológicas puede ser explicada por los dos primeros ejes (Tabla 6). En la Figura 4a se observa que las estaciones de la LMI (códigos negros), así como de la LME (azules) y de la LMA (lilas) tienen una tendencia de separación entre ellas.

En aquellas estaciones de la LMI y LME (lado derecho de la Figura 4a y 4b en negro y azul) se observa a las temperaturas y oxigeno disuelto (OD) relacionadas con la familia Chironomidae, Ephydridae, Oligoquetos y 
la presencia de Saldidae e Hydrophillidae en la LMI; Thiaridae y Chironomidae también relacionadas con estos 2 parámetros en la LME. En el otro extremo (lado izquierdo de la Figura 4a, en lila y 4b), en estaciones de la LMA, se observa que la conductividad eléctrica (CE) y profundidad se encuentran relacionadas con la familia Hydrobiidae y la presencia de Elmidae y Athericidae.

\section{Discusión}

Basados en los resultados arrojados por el análisis de Componentes principales, los parámetros fisicoquímicos que explican la dinámica de las 3 lagunas en los humedales de Ventanilla son el OD y CE. Mientras que al relacionar los parámetros fisicoquímicos y la comunidad de macroivertebrados bentónicos a través del análisis de Correspondencia Canónica, se observó sitios con presencia de familias como Chironomidae, Ephydridae y Oligochaeta y Thiaridae relacionadas con el OD y la temperatura del agua, y sitios predominados por Hydrobiidae relacionada con la CE.

En este estudio tanto en la LMI como en la LMA la especie dominante fue Hidrobia sp. (Hydrobiidae) adaptándose a aguas dulces como salobres, afectando la diversidad de las lagunas. Estudios similares registrados para la región sudeste y sur de Brasil, Montevideo y Buenos aires dicha especie también se encuentran habitando aguas con influencia marina, en el fondo areno-lodoso, en algas del genero Enteromorpha (Silva, 2003), ingiriendo la película bacteriana que se forma entre el material orgánico depositado. Así mismo, Rojas (2001) observó en el sector Río Limón-San Carlos, una comunidad representada por 20 especies, pertenecientes casi en su totalidad a los moluscos, lo que afecta la diversidad del lugar.

Chinchayán et al. (2008) señala que Hydrobia sp (prosobranquio) fue una de las especies de más baja presencia en los Humedales de Villa, lo que indicaría que no es característica de esas lagunas, asumiendo que es una especie introducida, sin embargo la información es escaza para Perú.

El taxón predominante en la LME fue Melanoides tuberculata (Thiaridae) en cuanto a abundancia (\%) se refiere, mientras que los demás taxones presentaron abundancias bajas, lo que afecto en gran manera la diversidad encontrada en las lagunas 1 y 9 . Iannacone (2003) señala como taxón dominante al gasterópodo $M$. tuberculata, responsable de la baja diversidad encontrada en las lagunas de Puerto Viejo, Lima.

M. tuberculata es un prosobranquio que fue introducido al Perú en la década del 70, posiblemente vía los acuarios y actualmente la distribución geográfica de esta especie se ha ampliado en los ecosistemas limnéticos, inclusive en la región Neártica y en el Neotrópico, ya que este thiarido es un caracol viajero, partenogenético, ovovivíparo y con longevidad máxima de cinco años (Albarran et al., 2009). Es un organismo principalmente detritívoro (Hutchinson, 1993) pero en determinadas condiciones, basadas en este estudio, se comporta como herbívoro, conformando su dieta de perifiton (algas, diatomeas y bacterias) y macrófitos que se encuentra en el sustrato (Pegado et al. 2007 y Cummins et al., 2005).

Vivar et al. (1998) en los humedales de Villa y Pegado et al. (2007) en el poso Sao Joao, rio Taperóa, Brasil, observaron a $M$. tuberculata semienterrados en sustrato limo-arenoso con la capacidad de cerrar el opérculo como una forma de adaptación, algunos individuos se encuentran en las raíces de las plantas emergentes y sumergentes, condición similar fue observada en la LME en estudio, con la presencia de totora en E5, Chara sp en E3 y E4. Albarran et al. (2009) observaron que en aguas con incremento de la salinidad representa una barrera abiótica al buen desarrollo de esta especie, este comportamiento es contrario al registrado por Roessler et al. (1977) quienes señalan que $M$. tuberculata puede subsistir en estuarios de Florida, Estados Unidos de Norteamérica.

El aumento de vegetación de orilla, del alga Chara sp (Charophyta) y microalgas en la LME se deben principalmente al aporte de detergente usado en la actividad de lavado por los pobladores en dicha laguna, aumentando los niveles de nitrato (ECA). En estas condiciones $M$. tuberculata se desarrolla muy bien en estas aguas. Estudios similares se observan en los pantanos de Centla, México, donde la abundancia de $M$. tuberculata tuvo una correlación significativa y directa con los nitratos (Albarran et al., 2009).

En cuanto a los quironómidos (Chironomus sp), relacionados con el OD, estuvieron presentes en la mayoría de las estaciones de la LMI y LME registrándose estadios inmaduros entre larvas y pupas, al igual que el humedal costero de las Albuferas de Medio Mundo, Lima (Iannacone et al., 2001) y las lagunas de Puerto viejo, Lima (Iannacone et al., 2003). Son organismos tolerantes, capaces de vivir en condiciones de anoxia por varias horas. Se alimenta de materia orgánica depositada en el sedimento, favoreciendo su adaptación a los más diversos ambientes, no posee ningún tipo de exigencia en cuanto a diversidad de hábitat y microhábitat refiere (Goulart et al., 2003). Los cuerpos de agua estudiados van de aguas dulces a salobres con detritus en el fondo, lo cual favorece a las larvas de Chironomus, quienes viven en los sedimentos como fango y arena pudiéndose encontrar en distintos tipos de agua, algunas viven en material vegetal en descomposición tolerando concentraciones bajas de oxígeno, por lo que los consideran indicadores de aguas contaminadas (aguas mesoeutróficas) (Cole, 1983; Roldán, 1992)

Por otro lado, Ephydridae y oligochaeta están relacionados con el OD y la temperatura del agua en la LMI. Según Ríos (2008), Ephydridae se caracteriza por habitar en aguas contaminadas, pudiendo ser indicadores de aguas con alto grado de contaminación, están adaptadas a desarrollarse en cuerpos de agua muy alcalinos o salinos. Los Oligoquetos estuvieron presente en los meses de abril a junio debido a la cantidad de detritus en descomposición que le sirve como alimento 
(Roldán, 1992), desapareciendo en los meses siguientes, que podría deberse al movimiento de las aguas del fondo (causadas por infiltración de agua dulce), removiendo el hábitat donde se encuentra y este cambio no solo implica un cambio físico de sustrato sino principalmente del recurso alimenticio. Según Roldán (1988), la mayoría de los oligoquetos viven en aguas eutróficas sobre fondo fangoso, con abundante cantidad de detritus y con concentraciones bajas de oxigeno.

En las tres lagunas en estudio la dominancia de taxones en relación con los demás es notoria presentando baja diversidad (0,042 - 2,6). Alonso et al. (2005) demuestra que las especies más abundantes y dominantes afectaban los valores de diversidad. Valores menores de 2,5 son indicativos que el ecosistema se encuentra sometido a tensión antrópica, presentando aguas contaminadas (Crisci, 1983).

La diversidad en este estudio es el resultado de algunos factores que producen estrés ambiental, como podría ser la fluctuación hidrológica que se produce debido al aumento del nivel del agua en las lagunas en los meses de mayo y junio, y el descanso del nivel del agua durante los meses de abril y parte mayo, aumentando y reduciendo drásticamente el volumen de los cuerpos de agua, por ende afectando a las comunidades de macroinvertebrados presentes. La presión antrópica es otro de los factores que inducen a estrés ambiental debido a la presencia de urbanización muy cercana a los humedales, modificando el hábitat e impactando en las comunidades macrobentónicas.

Este valor de diversidad nos puede indicar que no muchos organismos bentónicos están en capacidad de tolerar estas condiciones tan estresantes, y por el contrario, los Thiaridos e Hydrobidos pueden desarrollarse sin ningún problema cuando se enfrentan con estas condiciones.

Las características de la LMI son propicias para albergar a una gran diversidad de aves, aportando a la cadena trófica, en cuanto a materia orgánica (detritus animal) se refiere, con la presencia de Coliformes Totales en niveles altos afectando la conservación del ambiente acuática (Estándares de Calidad Ambiental ECA), en tanto, este hecho podría deberse a infiltraciones de la cuenca del río Chillón, que viene pasando por una crítica situación de contaminación.

Por otro lado, LME sufre presión antrópica y la población al no tener una red de servicio sanitario se ve en la necesidad de construir cilos provisionales, afectando e infiltrando con desechos fecales el ambiente acuático y en algunos casos el arrojo de basura, sumado a ello las infiltraciones de aguas del rio Chillón. La LMA también sufre presión antrópica por estar ubicada cerca al balneario Costa Azul observando la presencia de desperdicios en descomposición y basura flotando en el agua, este puede ser la causa de que en abril y parte de mayo el OD no esté de acorde con los niveles permisibles para la conservación del ambiente acuática (ECA), cambiando el panorama de mayo a junio por la subida del nivel del agua.

\section{Conclusiones}

La elevada abundancia de taxones tolerantes disminuye la influencia de taxones raros y sensibles en las tres lagunas en estudio. Los humedales de Ventanilla es un ecosistema que enfrenta actualmente problemas antrópicos y ambientales, al evaluar los componentes que estructuran la comunidad de macroinvertebrados bentónicos se observa muy baja diversidad, afectada por la dominancia de dos organismos, Hydrobia sp y $M$. tuberculata. Futuros trabajos en este ecosistema deberían de estar orientados a la evaluación de macrobentos a diferentes niveles de profundidad para poder tener una vista más amplia de la comunidad de macroinvertebrados bentónicos de los humedales de Ventanilla.

\section{Agradecimientos}

Las autoras agradecen a la Oficina Académica de Investigación de la UNALM por el financiamiento en etapa de gabinete. Por la colaboración de amigos y colegas Ana María Pisco, Elsa Mamani, Heidi Sánchez, María Salazar, María Sánchez, Pedro Sánchez, Sofia Challco y Susana Dávila, durante las salidas al campo, así como comentarios, críticas y sugerencias. A la Municipalidad de Ventanilla y la Región Callao por el permiso concedido para el ingreso al Humedal.

\section{Literatura citada}

Albarran, N.C.; Rangel, L. J. \& Gamboa, J. 2009. Distribución y abundancia de Melanoides tuberculata (Gastropoda: Thiaridae) en la Reserva de la Biosfera Pantanos de Centla. Tabasco. México. Acta Zoológica Mexicana. 25 (1): 93-104.

Alonso, A. \& Camargo, J. A. 2005. Estado actual y perspectivas en el empleo de la comunidad de macroinvertebrados bentónicos como indicadora del estado ecológico de los ecosistemas fluviales españoles. Ecosistemas. 14(3): 1-12.

Alvarez, C. \& Iannacone, J. 2008. Nuevos registros de aves en los humedales de Ventanilla, Callao, Perú. Biologist. 6(1): 68-71.

Anderson, M. 2001. A new method for non-parametric multivariate analysis of variance. Austral Ecology 26, 3246.

Borror, D.; De Long, D. \& Thriplehorn, C. 1995. An introduction to the study of insects. Saunders College Publishing. $6^{\text {th }}$ Edition. USA.

Chinchayán, V.; Pulido, G.; Oblitas, L. \& Peña, F. 2008. Análisis y determinación de macrozoobentos en la Laguna Marvilla de los Humedales de Villa, Chorrillos - Perú. Universidad Ricardo Palma. Fundación OESA. España.

Clarke, K. R. 1993. Non-parametric multivariate analysis of changes in community structure. Australian Journal of Ecology. 18: 117-143. 
Cole, G.A. 1983. Manual de limnología. $3^{\text {ra }}$ edición. Hemisferio Sur. Buenos Aires, Argentina.

Cowardin, L. M.; Carter, V.; Golet, F.C. \& Laroe, E.T. 1979. Classification of wetlands and deepwater habitats of the United States. U.S. Fish \& Wildlife Service Pub. Washington.

Cuadras, C. M. 2012. Nuevos Métodos de análisis multivariante. Barcelona, España.

Cummins, K. W.; Merrit, R. W. \& Andrade, P.C. 2005. The use of invertebrate functional groups to characterize ecosystem attributes in selected streams and rivers in south Brazil. Studies on Neotropical Fauna and Environment. 40(1): 69-89.

Crisci, J. V. 1983. Introducción a la teoría y práctica de la taxonomía numérica. OEA. Washington, D. C.

Espinoza, N.\& Morales, F. E. 2008. Macroinvertebrados bentónicos de la Laguna Las Peonías, Estado Zulia, Venezuela. Boletín del Centro de Investigaciones Biológicas. 42 (3): 345-363.

Franco, P. J.; Sulca, Q. L. \& Cáceres, M. C. 2000. Cyanophytas de los Humedales del Valle de Ite Tacna. Libro de Resúmenes del VIII Congreso Nacional de Botánica, Abril 24 - 28, 2000. Arequipa.: 51

Gittenberger, A. \& W. Van Loon. 2011. Common Marine Macrozoobenthos Species in The Netherlands, their Characteristics and Sensitivities to Environmental Pressures. GiMaRis report 2011.08. Netherlands.

Gopal, B.; Turner, R. E.; Wetzel, R. C. \& Whigham, D. F. 1982. Wetlands-Ecology and Manugement. National Institute of Ecology and International Scientific Publications, Jaipur.

Goulart, M. D \& Callisto, M. 2003. Bioindicadores de qualidade de água como ferramenta em estudos de impacto ambiental. Revista da FAPAM. 2 (1): 156-164.

Hutchinson, G. E 1993. Gastropods molluses. A treatise on Limnology Volume VI. The Zoobenthos. New York, USA.

Iannacone, J.; Alayo, M.; Arrascue, A.; Sánchez, J. y Abanto, M. 2001. Trampas de luz para evaluaciones rápidas de la biodiversidad de la antropofauna: análisis de tres casos. Wiñay Yachay. 5: 7-20.

Iannacone, J.; Mansilla, J. \& Ventura, K. 2003. Macroinvertebrados en las lagunas de Puerto Viejo, Lima - Perú. Ecología aplicada. 2 (1): 116-124

Moliner, E.A.\& González, A.C.2003. Recomendaciones para la toma de muestras de agua, biota y sedimentos en humedales de Rasar. Editorial Parques Nacionales.

Obando, C. M.; Campos, C. M.; García, Z. P \& Romero, N. M. 1998. Inventario de la diversidad ornitológica del humedal del caudato Pisco durante 1997. Ecología (Perú). 1: 72 - 75.
Pegado, F. C.; Leite de Melo, T.; Freitas, A. H.; Da Silva, H.; Targino de Oliveira, E.; Nóbrega, B. \& Dias, A. C. 2007. Macroinvertebrados bentônicos como bioindicadores de qualidade ambiental de corpos aquáticos da Caatinga. Oecologia Brasiliensis. 11(3):397409.

Pulido, V. 1998. La zona reservada de los Pantanos de Villa en el contexto de la conservación de los humedales en el Perú. En los Pantanos de Villa, Biología y Conservación. Museo de Historia Natural. UNMSM. Lima, Perú. Serie de divulgación. (11): 147-159.

Ríos, B. 2008. Comunidades de macroinvertebrados en un rio altoandino: Importancia del microhabitat, dinámica de la deriva, papel de la materia orgánica y relevancia de la ovoposición. Tesis doctoral. Universitat de Barcelona.

Rojas, J. 2001. Estructura de la comunidad macrobentónica del Sector Río Limón-Isla San Carlos, Estado Zulia, Venezuela. Tesis de Magíster Scientiarum. Universidad del Zulia. Maracaibo, Venezuela.

Roldán, G. 1988. Guía de estudio de macroinvertebrados acuáticos del departamento de Antioquía. Editorial Universidad de Antioquia. Medellín, Colombia.

G. 1992. Fundamentos de limnología Neotropical. Editorial Universidad de Antioquia. Medellín, Colombia. Roessler, M. A.; Beardsley, G. L \& Tabb, D. C. 1977. New records of the introduced snail, Melanoides tuberculata (Mollusca: Thiaridae) in south Florida. Florida Scientist. 40(1): 87-94.

Sadeghi , S.; Bagher, S.; Ahmad, V. \& Maryamabadi, A. 2010. Species Diversity of Macrobenthic Communities in Salakh Region, Qeshm Island, Iran. World Journal of Fish and Marine Sciences 2 (6): 539-544.

Silva, M. 2003. Hydrobiidae (Gastropoda, Neotaenioglossa, Rissoidea) da palnicie costeira deo Rio Grande do Sul. Tese doutoral em Biología Animal. Universidad Federal do Río Grande do Sul. Porto Alegre - Brasil.

Vivar, G.; Ramíres, M. \& Huaman, P. 1998. Moluscos de los Pantanos de Villa y su aporte a la conservación. Boletín del Museo de Historia Natural, UNMSM. Lima, Perú. Serie de divulgación. 11: 55-73. 


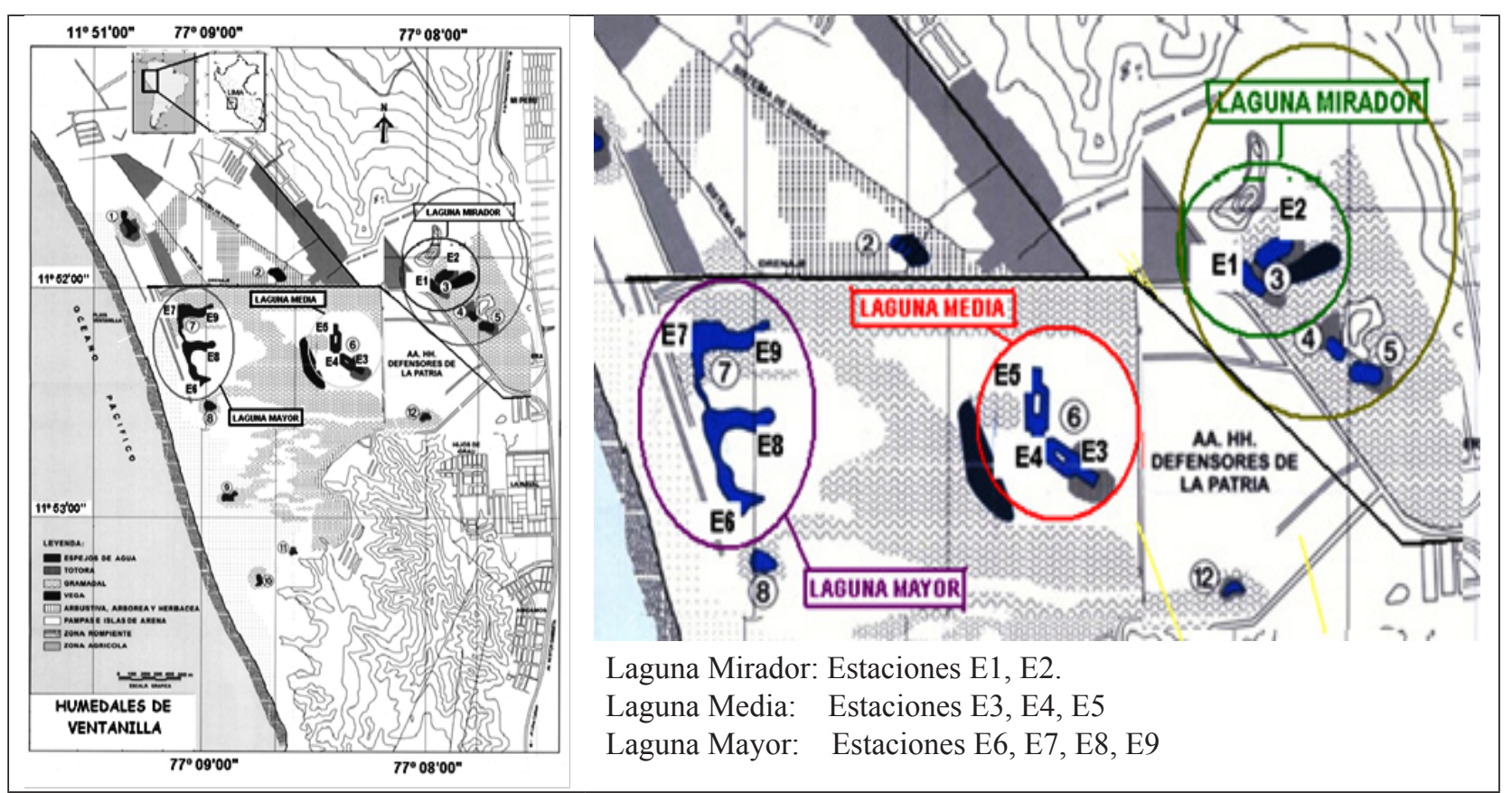

Figura 1. Ubicación de las 9 estaciones de muestreo en los Humedales de Ventanilla.

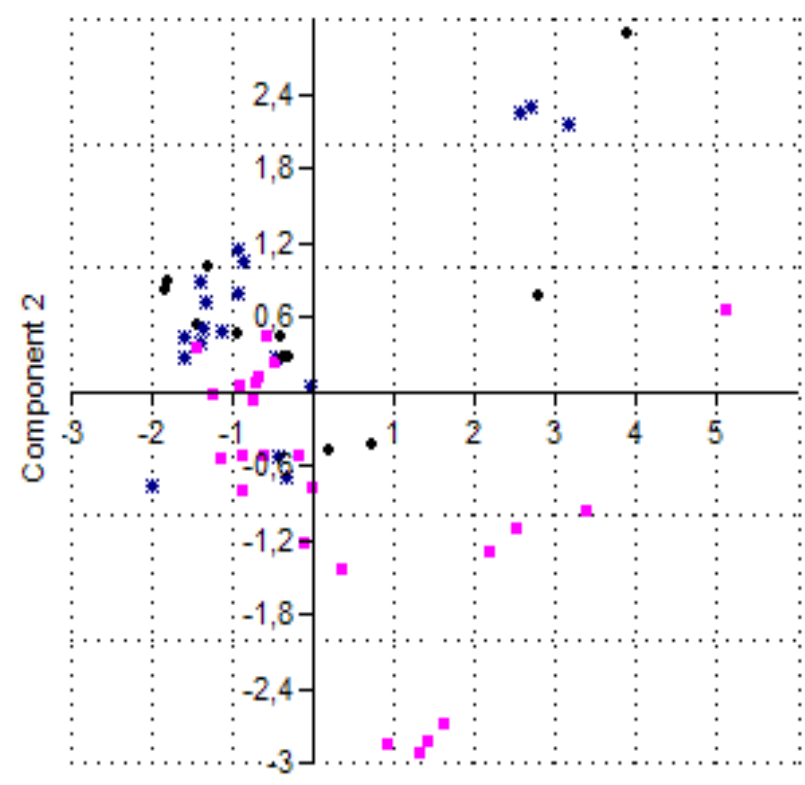

Component 1

(a)

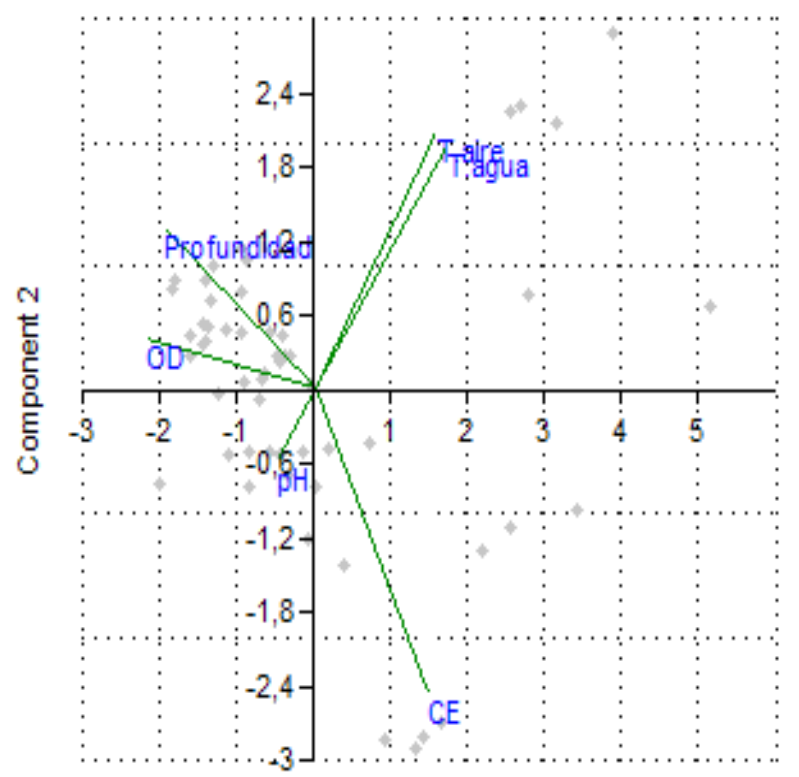

Component 1

(b)

Figura 2. Ordenamiento de las estaciones (a) según el Análisis de Componentes Principales de acuerdo a las variables fisicoquímicas (b). Los códigos negros indican estaciones de la LMI, los azules de la LME y los lilas de la LMA. 


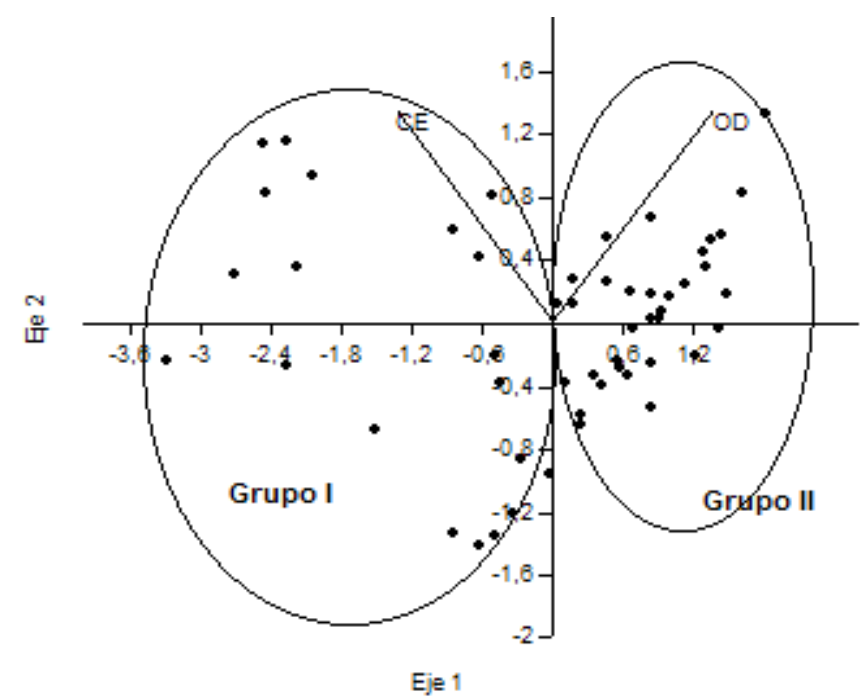

Figura 3. Análisis de componentes principales de la variacon de los factores fisicoquímicos en función de las estaciones y meses de muestreo de los Humedales de Ventanilla (Grupo 1: estaciones y meses con CE alta y OD bajas; Grupo II: estaciones y meses con OD alto y CE bajas).

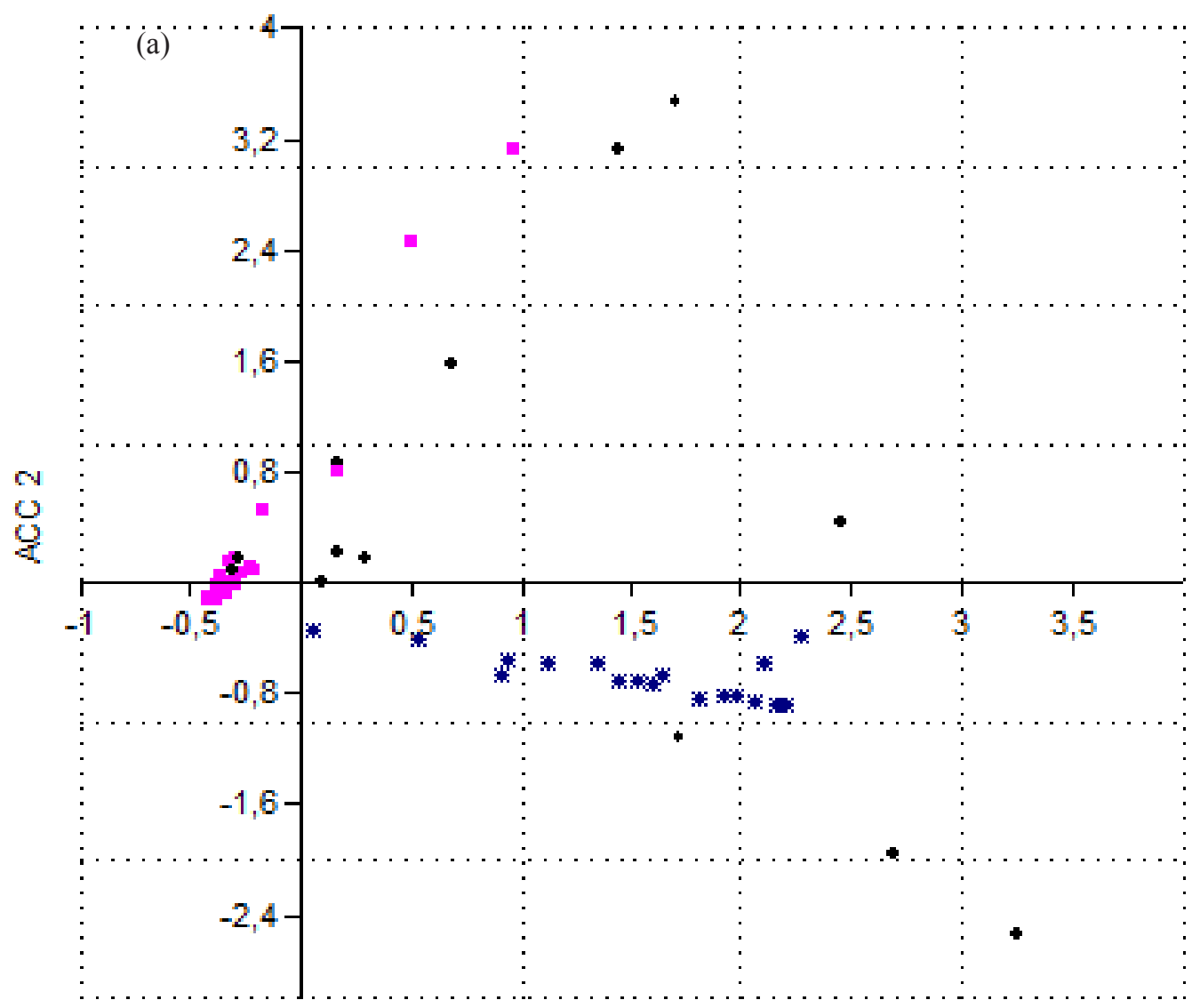

ACC 1

Figura 4. Resultado del Análisis de Correspondencia Canónica (ACC) en el ordenamiento de las estaciones muestreadas de los humedales de Ventanilla, Callao (a) según la abundancia de los macroinvertebrados bentónicos relacionado con los parámetros fisicoquímicos (b). Los códigos negros indican estaciones de la LMI, los azules de la LME y los lilas de la LMA. 


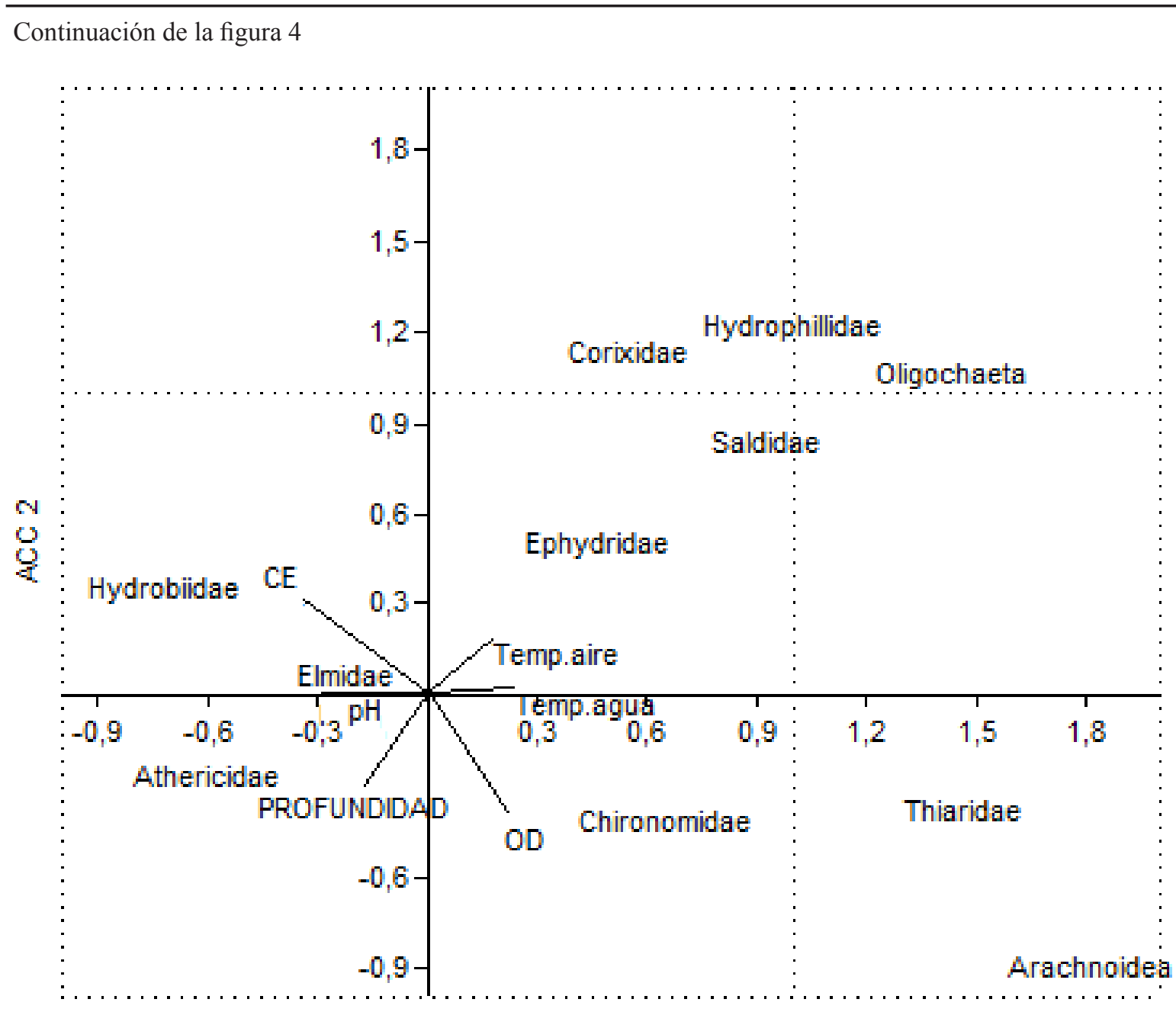

ACC 1

Tabla 1. Posición Geográfica de las nueve estaciones muestreadas en los Humedales de Ventanilla.

\begin{tabular}{ccc}
\hline Laguna & \multicolumn{2}{c}{ Coordenaadas } \\
\cline { 2 - 3 } & Latitud (S) & Longitud (O) \\
\hline $\begin{array}{c}\text { Laguna Mirador } \\
\text { (LMI) }\end{array}$ & $11^{\circ} 52^{\prime} 21.7^{\prime \prime}$ & $77^{\circ} 08^{\prime} 17.4^{\prime \prime}$ \\
E1 & $11^{\circ} 52^{\prime} 15.1^{\prime \prime}$ & $77^{\circ} 08^{\prime} 17.0^{\prime \prime}$ \\
E2 & & \\
Laguna Media & & \\
(LME) & $11^{\circ} 52.4^{\prime} 41.3^{\prime \prime}$ & $77^{\circ} 08^{\prime} 38.1^{\prime \prime}$ \\
E3 & $11^{\circ} 52^{\prime} 40^{\prime \prime}$ & $77^{\circ} 08^{\prime} 40.7^{\prime \prime}$ \\
E4 & $11^{\circ} 52^{\prime} 40.2^{\prime \prime}$ & $77^{\circ} 08^{\prime} 34.9^{\prime \prime}$ \\
E5 & & \\
Laguna Mayor & & \\
(LMA) & $11^{\circ} 52^{\prime} 41.1^{\prime \prime}$ & $77^{\circ} 09^{\prime} 10.3^{\prime \prime}$ \\
E6 & $1^{\circ} 52^{\prime} 25.0^{\prime \prime}$ & $77^{\circ} 09^{\prime} 16.6^{\prime \prime}$ \\
E7 & $11^{\circ} 52^{\prime} 36.4^{\prime \prime}$ & $77^{\circ} 09^{\prime} 11.9^{\prime \prime}$ \\
E8 & $1^{\circ} 52^{\prime} 32.3^{\prime \prime}$ & $77^{\circ} 09^{\prime} 08.7^{\prime \prime}$ \\
E9 &
\end{tabular}

Tabla 2. Matriz de entre los parámetros fisicoquímicos de la Ordenación de Componentes Principales.

\begin{tabular}{lcc}
$\begin{array}{c}\text { Componentes } \\
(\text { Ejes })\end{array}$ & $\begin{array}{c}\text { Valores propios } \\
\text { (Eigenvalue) }\end{array}$ & $\begin{array}{c}\text { Varianza } \\
(\%)\end{array}$ \\
\hline OD $(\mathrm{mg} / \mathrm{l})$ & 2,76 & 46,00 \\
$\mathrm{CE}(\mathrm{mS})$ & 1,44 & 24,02 \\
$\mathrm{pH}$ & 1,06 & 17,61 \\
$\mathrm{Temp}$, aire $\left(\mathrm{C}^{\circ}\right)$ & 0,29 & 4,81 \\
Profundidad $(\mathrm{cm})$ & 0,26 & 4,40 \\
Temp, agua $\left(\mathrm{C}^{\circ}\right)$ & 0,19 & 3,16 \\
\hline OD $=$ Oxigeno disuelto $\mathrm{CE}=$ Conductividad & \\
& & \\
& &
\end{tabular}


Tabla 3. Parámetros fisicoquímicos y microbiológicos de tres lagunas durante los meses de muestreo, en los Humedales de Ventanilla, Callao.

\begin{tabular}{|c|c|c|c|c|c|c|c|c|c|}
\hline Mes & Laguna & $\begin{array}{l}\mathrm{T}^{\circ} \mathrm{C} \\
\text { aire }\end{array}$ & $\begin{array}{l}\mathrm{T}^{\circ} \mathrm{C} \\
\text { agua }\end{array}$ & $\begin{array}{c}\mathrm{OD} \\
(\mathrm{mg} / \mathrm{L})\end{array}$ & $\mathrm{pH}$ & $\mathrm{CE}(\mathrm{mS})$ & $\begin{array}{l}\text { Nitratos } \\
(\mathrm{mg} / \mathrm{L})\end{array}$ & $\mathrm{CT}(\mathrm{NMP} / 100 \mathrm{~mL})$ & $\begin{array}{l}\text { Profundidad } \\
\text { (cm) }\end{array}$ \\
\hline \multirow{3}{*}{ Abr. } & LMI & 29,5 & 24,0 & 2,5 & 6,8 & 27,4 & 0,1 & 79000 & 23,0 \\
\hline & LME & 25,0 & 29,3 & 3,0 & 7,2 & 13,5 & 22,2 & 1700 & 27,3 \\
\hline & LMA & 22,0 & 24,8 & 2,0 & 7,4 & 61,0 & 0,1 & $<1,8$ & 15,0 \\
\hline \multirow{3}{*}{ May. } & LMI & 17,0 & 18,5 & 4,8 & 6,8 & 22,2 & - & - & 26,5 \\
\hline & LME & 16,3 & 18,7 & 5,7 & 7,2 & 14,8 & - & - & 31,0 \\
\hline & LMA & 15,6 & 17,3 & 3,8 & 7,3 & 70,1 & - & - & 17,5 \\
\hline \multirow{3}{*}{ Jun. } & LMI & 17,3 & 20,0 & 8,5 & 6,8 & 5,9 & 0,1 & 11000 & 43,5 \\
\hline & LME & 16,5 & 18,0 & 7,8 & 7,2 & 9,0 & 23,1 & 13000 & 46,3 \\
\hline & LMA & 17,1 & 17,5 & 6,1 & 7,3 & 37,5 & 0,1 & 130 & 36,0 \\
\hline \multirow{3}{*}{ Jul. } & LMI & 16,3 & 20,3 & 6,8 & 6,8 & 17,2 & - & - & 51,0 \\
\hline & LME & 18,3 & 21,0 & 7,7 & 7,2 & 13,8 & - & - & 56,7 \\
\hline & LMA & 16,8 & 18,8 & 5,1 & 7,3 & 21,3 & - & - & 52,5 \\
\hline \multirow{3}{*}{ Ago. } & LMI & 20,3 & 17,8 & 7,0 & 7,5 & 12,7 & - & - & 58,5 \\
\hline & LME & 20,8 & 17,0 & 7,4 & 7,6 & 13,5 & - & - & 65,7 \\
\hline & LMA & 18,0 & 17,5 & 6,4 & 7,4 & 25,3 & - & - & 57,5 \\
\hline \multirow{3}{*}{ Set. } & LMI & 16,8 & 19,0 & 6,7 & 7,0 & 11,5 & 15,5 & 7900 & 70,0 \\
\hline & LME & 17,2 & 20,5 & 6,4 & 7,6 & 13,3 & 9,79 & 1700 & 67,3 \\
\hline & LMA & 17,8 & 19,0 & 5,7 & 7,5 & 14,2 & 10,5 & 1300 & 59,5 \\
\hline
\end{tabular}

LMI = Laguna Mirador, LME = Laguna Media, LMA = Laguna Mayor, CT = Coliformes Totales.

Tabla 4. Abundancia promedio mensual de los macroinvertebrados bentónicos encontrados en las 3 lagunas de los Humedales de Ventanilla, Callao.

\begin{tabular}{|c|c|c|c|c|c|c|c|c|}
\hline \multirow{2}{*}{$\begin{array}{l}\text { Laguna Mirador (LMI) } \\
\text { Taxon }\end{array}$} & \multicolumn{6}{|c|}{ Mes de muestreo } & \multirow{2}{*}{$\begin{array}{l}\text { Abun. } \\
\text { Prom. }\end{array}$} & \multirow{2}{*}{$\frac{\text { Abun. }}{(\%)}$} \\
\hline & Abr & May & Jun & Jul & Ago & Set & & \\
\hline Oligochaeta & 157 & 287 & 64 & 0 & 0 & 0 & 84.7 & 10.6 \\
\hline Arachnoidea & 11 & 3 & 387 & 163 & 108 & 3 & 112.5 & 14.1 \\
\hline Elmidae & 0 & 2 & 0 & 0 & 0 & 0 & 0.3 & 0.04 \\
\hline Hydrophillidae & 166 & 317 & 0 & 27 & 12 & 4 & 87.7 & 11 \\
\hline Chironomus sp & 15 & 24 & 15 & 65 & 35 & 2 & 26.0 & 3.3 \\
\hline Ephydridae & 197 & 243 & 35 & 72 & 47 & 41 & 105.8 & 13.3 \\
\hline Centrocorisa sp & 98 & 120 & 0 & 0 & 0 & 0 & 36.3 & 4.6 \\
\hline Saldidae & 20 & 38 & 0 & 10 & 29 & 8 & 17.5 & 2.2 \\
\hline Hydrobia sp. & 187 & 660 & 10 & 288 & 306 & 392 & 307.2 & 38.6 \\
\hline Melanoides tuberculata & 8 & 8 & 10 & 13 & 24 & 49 & 18.7 & 2.3 \\
\hline A.P. de ind. por muestreo & 85.9 & 170 & 52.1 & 63.8 & 56.1 & 49.9 & & \\
\hline Laguna Media (LME) & & & $\mathrm{s}$ de $\mathrm{m}$ & treo & & & Abun. & Abun. \\
\hline Taxon & Abr & May & Jun & Jul & Ago & Set & Prom. & $(\%)$ \\
\hline Oligochaeta & 11 & 0 & 0 & 0 & 0 & 0 & 1.8 & 0.2 \\
\hline Arachnoidea & 9 & 2 & 15 & 6 & 4 & 0 & 6.0 & 0.8 \\
\hline Elmidae & 0 & 1 & 0 & 0 & 0 & 0 & 0.2 & 0.02 \\
\hline Hydrophillidae & 0 & 4 & 0 & 4 & 0 & 0 & 1.3 & 0.17 \\
\hline Chironomus sp & 5 & 17 & 46 & 147 & 95 & 73 & 63.8 & 8.3 \\
\hline Ephydridae & 0 & 2 & 9 & 3 & 1 & 0 & 2.5 & 0.3 \\
\hline Centrocorisa sp & 0 & 1 & 0 & 0 & 0 & 0 & 0.2 & 0.02 \\
\hline
\end{tabular}


Continuación de tabla

\begin{tabular}{|c|c|c|c|c|c|c|c|c|}
\hline Hydrobia sp. & 104 & 113 & 87 & 775 & 192 & 338 & 268.2 & 34.9 \\
\hline Melanoides tuberculata & 385 & 415 & 186 & 987 & 360 & 208 & 423.5 & 55.2 \\
\hline A.P. de ind. por muestreo & 57.1 & 61.7 & 6.11 & 214 & 72.4 & 68.8 & & \\
\hline Laguna Mayor (LMA) & \multicolumn{6}{|c|}{ Mes de muestreo } & Abun. & Abun. \\
\hline Taxon & Abr & May & Jun & Jul & Ago & Set & Prom. & $(\%)$ \\
\hline Arachnoidea & 0 & 2 & 9 & 16 & 2 & 1 & 5.0 & 0.09 \\
\hline Elmidae & 0 & 0 & 25 & 0 & 0 & 0 & 4.2 & 0.08 \\
\hline Hydrophillidae & 32 & 2 & 10 & 0 & 0 & 0 & 7.3 & 0.13 \\
\hline Atherix sp & 0 & 8 & 12 & 0 & 4 & 7 & 5.2 & 0.09 \\
\hline Chironomus sp & 0 & 2 & 585 & 365 & 125 & 14 & 181.8 & 3.3 \\
\hline Ephydridae & 135 & 83 & 202 & 380 & 232 & 101 & 188.8 & 3.5 \\
\hline Centrocorisa sp & 135 & 76 & 67 & 41 & 0 & 32 & 58.5 & 1.07 \\
\hline Saldidae & 5 & 0 & 1 & 0 & 2 & 0 & 1.3 & 0.02 \\
\hline Hydrobia sp. & 1852 & 4858 & 10178 & 7990 & 2517 & 2587 & 4997 & 91.7 \\
\hline A.P. de ind. por muestreo & 240 & 559 & 1232 & 977 & 320 & 305 & & \\
\hline Abun. Prom. Total & 128 & 263 & 430 & 418 & 149 & 141 & & \\
\hline
\end{tabular}

A.P. de ind. =Abundancia promedio de individuos

Tabla 5. Valores de Diversidad (H'), Riqueza (d), Equidad (J') y Dominancia (D) en las estaciones de muestreo de 3 lagunas de los Humedales de Ventanilla, Callao.

\begin{tabular}{|c|c|c|c|c|c|c|c|c|c|}
\hline \multirow{2}{*}{ 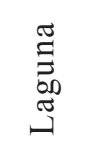 } & \multirow{2}{*}{ 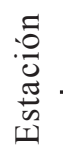 } & \multicolumn{2}{|c|}{$\begin{array}{l}\text { Diversidad Shannon- } \\
\text { Wiener (H') }\end{array}$} & \multicolumn{2}{|c|}{ Riqueza Margalef (d) } & \multicolumn{2}{|c|}{ Pielou's evenness (J') } & \multicolumn{2}{|c|}{$\begin{array}{l}\text { Dominancia de } \\
\text { Simpson (D) }\end{array}$} \\
\hline & & Rango & Media & Rango & Media & Rango & Media & Rango & Media \\
\hline \multirow{2}{*}{ LMI } & E1 & $1,17-2,65$ & 1.9 & $0,77-1,48$ & 1.1 & $0,42-0,8$ & 0.6 & $0,2-0,61$ & 0.4 \\
\hline & E2 & $0,50-2,0$ & 1.3 & $0,26-0,99$ & 0.6 & $0,27-0,82$ & 0.6 & $0,23-0,84$ & 0.5 \\
\hline \multirow{3}{*}{ LME } & E3 & $0,55-1,5$ & 1.1 & $0,23-0,78$ & 0.5 & $0,56-0,82$ & 0.7 & $0,41-0,61$ & 0.6 \\
\hline & E4 & $0,7-1,78$ & 1.1 & $0,17-0,88$ & 0.4 & $0,57-0,76$ & 0.7 & $0,35-0,69$ & 0.5 \\
\hline & E5 & $1,33-1,91$ & 1.6 & $0,57-1,11$ & 0.9 & $0,64-0,74$ & 0.7 & $0,31-0,45$ & 0.4 \\
\hline \multirow{4}{*}{ LMA } & E6 & $0,17-2,0$ & 0.9 & $0,25-0,88$ & 0.6 & $0,07-0,86$ & 0.4 & $0,27-0,96$ & 0.7 \\
\hline & E7 & $0,11-1,03$ & 0.6 & $0,26-1,16$ & 0.6 & $0,05-0,5$ & 0.3 & $0,63-0,97$ & 0.8 \\
\hline & E8 & $0,04-1,41$ & 0.5 & $0,28-0,54$ & 0.4 & $0,02-0,89$ & 0.3 & $0,4-0,99$ & 0.8 \\
\hline & E9 & $0,41-1,1$ & 0.8 & $0,29-0,86$ & 0.6 & $0,23-0,47$ & 0.3 & $0,6-0,87$ & 0.7 \\
\hline
\end{tabular}

Tabla 6. Valores propios y varianza de los ejes formados por el Análisis de Correspondencia Canónica (ACC).

\begin{tabular}{ccc}
\hline Ejes & $\begin{array}{c}\text { Valores propios } \\
\text { (Eigenvalue) }\end{array}$ & $(\%)$ \\
\hline 1 & 0.21 & 64.18 \\
2 & 0.09 & 28.12 \\
3 & 0.021 & 6.26 \\
4 & 0.003 & 0.90 \\
5 & 0.002 & 0.53 \\
6 & 0.00001 & 0.002 \\
\hline
\end{tabular}

